\title{
Polymyalgia Rheumatica: A Challenging Diagnosis in Elderly Patients - Case Reports
}

\author{
Il-Kyu Im, Du Hwan Kim \\ Department of Rehabilitation Medicine, Dongsan Medical Center, School of Medicine, Keimyung University, Daegu, Korea
}

Corresponding Author: Du Hwan Kim, MD Department of Rehabilitation Medicine, Dongsan Medical Center, School of Medicine, Keimyung University, 56 Dalseong-ro, Jung-gu, Daegu

41931, Korea

Tel: $+82-53-250-7477$

Fax: +82-53-250-7205

E-mail: ripheonix@dsmc.or.kr

Received: July 27, 2017

Revised: October 12, 2017

Accepted: October 17, 2017
Polymyalgia rheumatica (PMR) is often characterized by bilateral shoulder pain and can be accompanied by giant cell arteritis (GCA). Here we report 2 cases in which a PMR diagnosis was made. A 61-year-old man with a 1-month history of gait disturbance and bilateral shoulder pain after an infarct in the left cerebellum. Fluorodeoxyglucose positron emission tomography/computed tomography (PET/CT) suggested PMR with GCA. A 78-year-old woman presented with alternating bilateral shoulder pain. She was repeatedly misdiagnosed with septic arthritis and treated with surgery. Based on clinical manifestations and laboratory tests, we retrospectively diagnosed her with PMR. We conclude that a careful analysis of the patient's history and a meticulous examination is necessary for the correct diagnosis of PMR in elderly patients.

Key Words: Polymyalgia Rheumatica, Shoulder Pain, Stroke

\section{INTRODUCTION}

Polymyalgia rheumatica (PMR) is an inflammatory condition that commonly involves the shoulders and/or the pelvic girdle in elderly individuals ${ }^{1)}$. The clinical features of PMR are similar to those of common shoulder diseases, such as frozen shoulder, rotator cuff tear with bursitis, and acute calcific tendinitis. Because of a significant increase in acutephase reactants (APR) and imaging findings suggesting infection, PMR may be confused with septic arthritis ${ }^{2)}$. Giant-cell arteritis (GCA) is an inflammatory vasculitis that typically occurs in medium and large arteries and may cause ischemic events $^{1)}$. PMR and GCA may occur simultaneously or separately". Some patients with PMR tend to develop GCA during the follow-up period. PMR can affect the musculoskeletal system because of periarticular inflammation and lesions in the nervous system that are secondary to ischemic events.

Both GCA and PMR incidence increases with age after 50 years old and it peaks at 70 to 80 years old ${ }^{1)}$. Although PMR and/or GCA are not commonly encountered disorders, physicians should be able to differentiate them from more common pain disorders and recognize them as risk factors for ischemic events of unknown origin.

We report one patient with PMR and GCA who was initially diagnosed with a stroke of unknown origin and another pati- ent with PMR mimicking bilateral septic arthritis who was treated with surgical debridement.

\section{CASE REPORTS}

\section{Case 1}

We obtained informed permission for the publication from this patient.. A 61-year-old man visited our outpatient clinic with gait disturbances due to bilateral posterior thigh pain, easy fatigue in both legs, and new onset headache after left inferior cerebellar infarction 1 month ago with no evidence of vas- culitis on magnetic resonance angiography (Fig. 1A). There were no severe disabling neurological sequelae except for mild ataxia after the stroke. He did not have any common risk factors (hypertension, diabetes, high cholesterol, smoking, or heart disease) for ischemic stroke.

On physical examination, a mild ataxic gait and painful, global stiffness without spasticity in both hip joints were observed. Plain radiography of the hip joints revealed no abnormal findings. Initially, we thought that these symptoms might be vague pain related to the stroke because the lumbar spine magnetic resonance imaging (MRI) and an electrodiagnostic studies did not demonstrate compressive lesions of the lumbar spine or peripheral nerve lesions. 
However, 2 weeks later, he complained that his symptoms became too severe to walk in the morning and he developed shoulder pain on both sides.

We reviewed his laboratory tests from the onset of his stroke. The first laboratory tests at admission revealed a markedly increased erythrocyte sedimentation rate $(120 \mathrm{~mm} /$ $\mathrm{hr})$ and C-reactive protein (CRP) $(6.68 \mathrm{mg} / \mathrm{dL})$. Because we strongly suspected rheumatic disease, we ordered further laboratory tests including antinuclear antibodies, rheumatoid factors (RF), anticyclic citrullinated peptide (anti-CCP) antibody, HLA B27, and thyroid function tests but the results did not reveal any abnormalities. Because of the bilateral shoulder and hip pain, markedly elevated APR, negative RF and anti-CCP, and history of stroke, we suspected PMR with GCA and evaluated the involvement of the large vessels by using ${ }^{18} \mathrm{~F}$-fluorodeoxyglucose (FDG) positron emission tomography/computed tomography (PET/CT), which revealed increased uptake along the walls of the large vessels (including the aorta, both subclavian arteries, the internal carotid artery, the femoral artery, and the vertebral artery) as well as in both shoulder and hip joints (Fig. 1B). Neck carotid CT angiography demonstrated concentric wall thickening in the descending aorta, aortic arch, and subclavian arteries (Fig. 1C). Based on clinical findings, laboratory tests, and imaging studies, we diagnosed him with PMR with GCA. In retrospect, his posterior thigh pain and global stiffness of
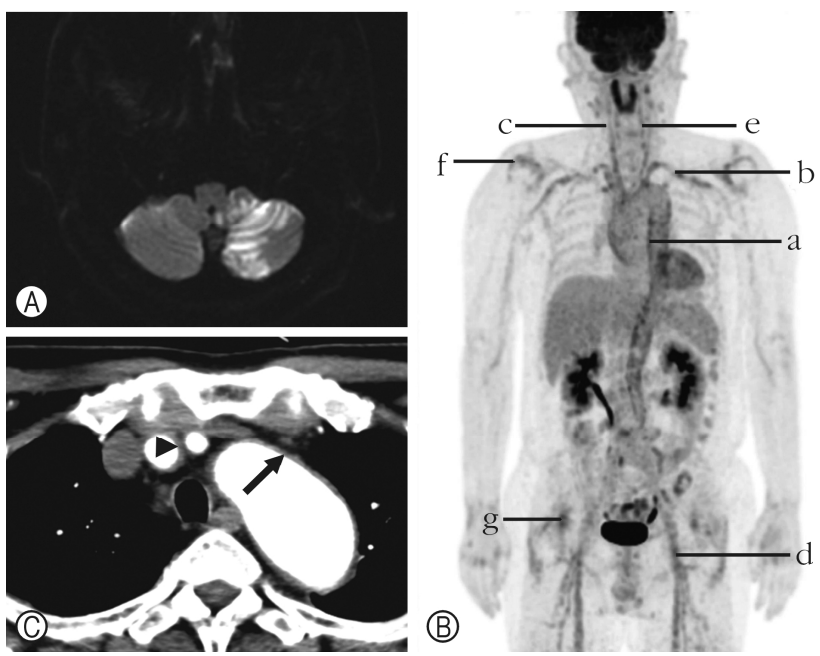

Fig. 1. (A) Diffusion-weighted image revealed acute ischemia in the left inferior cerebellum. (B) ${ }^{18} \mathrm{~F}$-fluorodeoxyglucose positron emission tomography/computed tomography revealed increased uptake along the walls of the large vessels (including the aorta [a], both subclavian arteries [b], the internal carotid artery [c], the femoral artery [d], the and vertebral artery [e]) as well as both shoulders (f) and hip (g) joints. (C) Neck carotid computed tomography angiography demonstrated concentric wall thickening in the brachiocephalic artery (arrowhead) and aortic arch (arrow). both hip joints were polymyalgic symptoms and we believe that his stroke might have been related to his GCA. Symptoms immediately resolved after treatment with intravenous methylprednisolone ( $500 \mathrm{mg} /$ day for 3 days) followed by a tapering of oral prednisolone (maintenance dose $5 \mathrm{mg} /$ day).

\section{Case 2}

We obtained informed permission for the publication from this patient.. A 73-year-old female presented with a 1-week history of severe neck pain and difficulty with neck rotation. On physical examination, there was prominent focal tenderness on the C7 spinous process and others were nonspecific. Cervical MRI with contrast enhancement showed a small amount of fluid collection over the $\mathrm{C} 6$ and $\mathrm{C} 7$ spinous processes with rim enhancement, suggesting cervical interspinous bursitis (Fig. 2A). History taking revealed that she had arthroscopic surgeries for septic arthritis 40 days ago on the right shoulder and one year ago on the left shoulder.

We retrospectively reviewed her medical records at the time of shoulder surgeries. One year ago, she was referred to our Emergency Department from other hospital because of poor oral intake, continuous fever for 3 days, neck stiffness, and left shoulder pain. Laboratory tests revealed no leukocytosis $\left(7,500 / \mathrm{mm}^{3}\right)$ and increased CRP $(11.97 \mathrm{mg} / \mathrm{dL})$. Tests for $R F$, anti-CCP, and antinuclear antibodies were negative. $A$ left shoulder MRI revealed diffuse enhancement of the subacromial and subdeltoid bursae and the glenohumeral joint synovium (Fig. 2B). An orthopedic surgeon diagnosed her with septic arthritis and decided to perform an exploratory arthroscopic operation including synovectomy and bursectomy with debridement. Arthroscopy showed joint effusion and a severely inflamed synovium without pus discharge (Fig. 2C). There was no sign of infection in the synovial fluid analysis and culture. Ten days after surgery, she complained of left wrist pain with swelling. Wrist MRI showed synovial thickening with enhancement, suggesting inflammatory arthropathy (Fig. 2D). At that time, her symptoms did not meet the criteria for rheumatoid arthritis. Her symptoms subsided after low-dose steroid therapy (methylprednisolone $5 \mathrm{mg} /$ day for 8 months) recommended by a rheumatologist. Forty days ago, she visited an orthopedic surgeon because of right shoulder pain without fever. The results of laboratory tests were similar to the previous results and a right shoulder MRI demonstrated findings similar to those of the previous left shoulder MRI (Fig. 2E). She received the same diagnosis and surgical treatment (Fig. 2F). In spite of the bilateral shoulder surgery and antibiotic treatment, moderate shoulder pain persisted (visual analogue scale 6 to 4).

Based on bilateral shoulder pain, peripheral joint and spinous process involvement, and laboratory results, we diagnosed her with PMR. Her remaining shoulder and neck pain 
improved within a few days after treatment with prednisolone (15 mg/day). She was followed postoperatively for 2 years and her symptoms were tolerable with prednisolone (10 mg/day).

\section{DISCUSSION}

We described a patient diagnosed with PMR with GCA, who initially presented with cerebellar infarct (without having any common risk factors for stroke) and whose diagnosis was delayed, and a patient diagnosed with PMR, who was initially misdiagnosed as having bilateral septic arthritis and was treated surgically.

Because PMR may have multiple manifestations, even patients with classic symptoms often experience a delay in diagnosis and management. A recent study reported that the interval between the onset and diagnosis was $13 \pm 13$ months ${ }^{2)}$. Because constitutional symptoms, such as general weakness, fever, weight loss, and high APR levels, are frequently observed in PMR, infection and concomitant cancer should also be considered $^{2}$. In clinical practice, PMR is commonly misdiagnosed as other diseases.

Although the majority of ischemic strokes are caused by large-artery atherosclerosis, cardioembolism, and cerebral small-vessel disease, vasculitis is also known to be a less common cause of ischemic stroke. GCA is known to increase the risk of transient ischemic accidents by $6 \%$ and that of stroke by $3 \% \%^{3)}$. Vertebrobasilar territory involvement in GCA is more frequent than in atherosclerotic stroke ${ }^{3)}$. Kang et al. ${ }^{4)}$ reported that apparently isolated PMR also significantly increased the risk of stroke. In elderly patients, it is difficult to clarify whether the stroke is related to PMR and/or GCA, or to thromboembolic or atherosclerotic vascular diseases. However, physicians should be aware of this entity as a cause of stroke because immediate therapy and secondary prevention can be different. Physicians should consider PMR and/or GCA in patients who have no vascular risk factors and complain of fatigue and shoulder pain after stroke.

It is inconclusive whether the occurrence of the stroke in case 1 was related to GCA. Because of the frequent occurrence of strokes in the elderly population, it is difficult to decide whether the ischemic event was related to GCA or to atherosclerotic or embolic vascular disease. GCA has multiple clinical manifestations, including fever, fatigue, weight loss, headache, jaw claudication, and visual loss ${ }^{5}$. In case 1 , only fatigue and vague new onset headache after stroke were present. In general, GCA patients with typical cranial symptoms have a high risk of developing ischemic events ${ }^{6,7}$. However, one study reported that ischemic events had occurred in GCA patients who had no other cranial presentations ${ }^{8)}$. Polymyalgic symptoms may start after the GCA ischemic
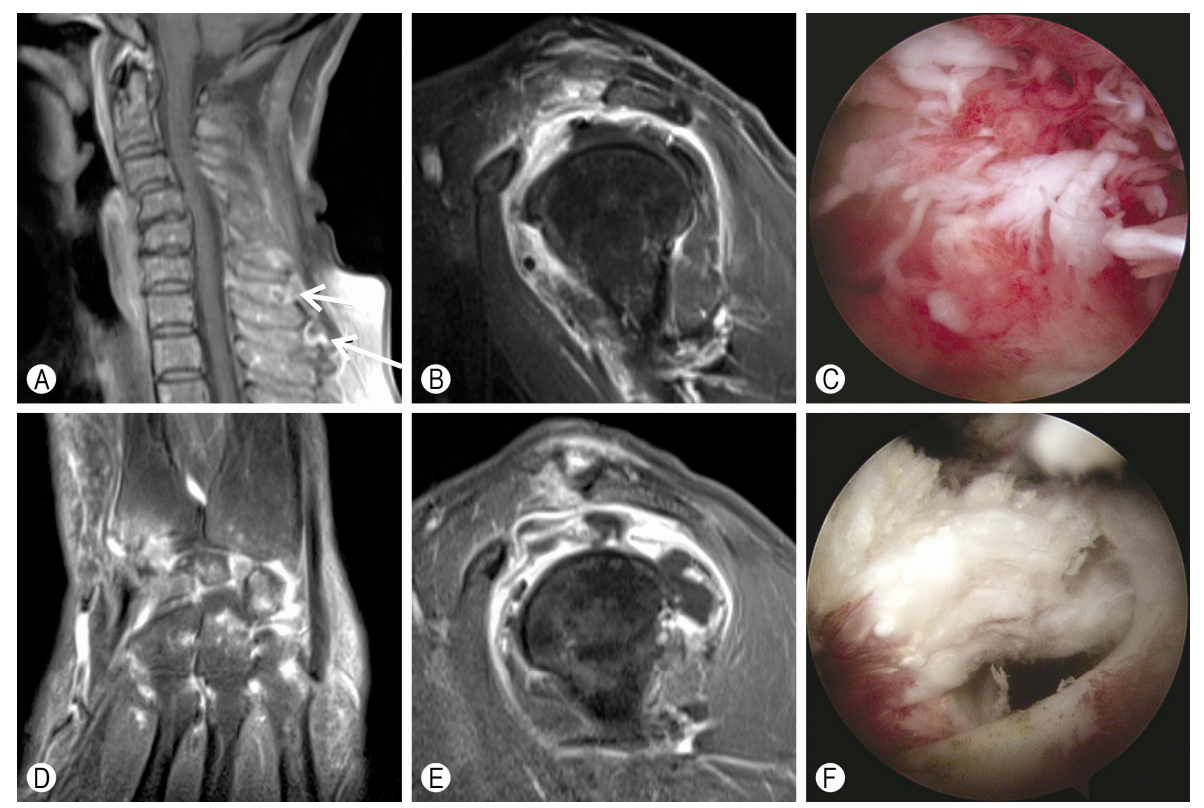

Fig. 2. (A) Cervical magnetic resonance imaging (MRI) showed a small fluid collection over the C6 and C7 spinous processes with rim enhancement (arrows). (B) Left shoulder MRI revealed diffuse enhancement of the subacromial and subdeltoid bursae and the glenohumeral joint synovium. (C) Arthroscopy of the left shoulder showed a severely inflamed synovium. (D) Wrist MRI showed synovial thickening with enhancement. (E) Right shoulder MRI revealed diffuse enhancement of the subacromial and subdeltoid bursae and the glenohumeral joint synovium. (F) Arthroscopy of the right shoulder showed a severely hyperemic synovium with fraying of the supraspinatus tendon. 
manifestation ${ }^{8)}$. Although it is difficult to conclude that the stroke in case 1 was related to GCA, the unexplained elevation of $A P R$, the absence of common risk factors for ischemic stroke, the area of the stroke (vertebrobasilar territory), and the bilateral shoulder and hip pain suggested that the stroke in case 1 was related to GCA/PMR.

The diagnosis of GCA/PMR depends on the presence of a combination of clinical findings. There is no specific test for GCA/PMR. PET/CT is a valuable tool for diagnosing GCA/PMR and differentiating from other diseases. PET/CT on PMR patients reveals a characteristic FDG uptake on the ischial tuberosity, greater trochanter, lumbar or cervical spinous processes, and scapulohumeral joint bursae ${ }^{9)}$. Yamashita et al. ${ }^{10)}$ found that the increase in FDG uptake at 2 or 3 lesions of the ischial tuberosity, greater trochanter, or lumbar spinous process had a high sensitivity and specificity, $85.7 \%$ and 88.2\% respectively, in patients with PMR compared with a control group that included rheumatoid arthritis patients.

In patients with suspected GCA, histologic analysis of a temporal artery biopsy has been regarded as the standard for diagnosis"). Although, temporal artery biopsy is less sensitive in diagnosing GCA, and GCA cannot be excluded even if the biopsy is negative. PET/CT is also a useful diagnostic tool for evaluating GCA. PET/CT is known to have a sensitivity of $89.5 \%$ and a specificity of $97.7 \%$ in GCA diagnoses ${ }^{111}$. Typically, the diagnosis of GCA was based on the presence of high FDG uptake in the vascular wall of the aorta, the supra-aortic branches, and the vertebral, iliac, and femoral arteries ${ }^{11)}$. In GCA patients, CT angiography has proven to be effective in showing aortitis, which is characterized by thickening of the vessel wall, inflammatory periaortic soft tissue change, stenosis, or aneurysm ${ }^{111}$.

Shoulder pain is a common problem in the elderly. Adhesive capsulitis, rotator cuff disease, and osteoarthritis are main causes of shoulder pain ${ }^{12)}$. Although septic arthritis of the shoulder is a relatively rare disease, it has recently increased in prevalence due to an increase in life expectancy, various shoulder problem, local injection on shoulder region, and immunocompromised conditions ${ }^{13)}$. The clinical manifestations of septic arthritis, such as shoulder pain with stiffness and fever, and elevated APR, can mimic PMR. Bone erosion with bone edema, synovial edema, and soft tissue edema on MRI, suggestive of septic arthritis, are not specific findings. Extracapsular inflammatory changes outside the joint cavity are common in PMR and can often be confused with a septic joint ${ }^{(4)}$. Dalkılıç et al. ${ }^{2)}$ reported that $30 \%$ of patients with PMR were given antibiotics before the correct diagnosis was made. The lack of awareness of PMR among physicians and the difficulty of differential diagnosis between PMR and septic shoulders may lead to unnecessary surgical treatment. In case 2, there were differences in clinical features from those of typical septic arthritis: the patient had no leukocytosis in spite of a high APR and a high-grade fever. Case 2 suggests that both PMR and septic arthritis should be considered as possible diagnoses if laboratory testing reveals markedly elevated APR without leukocytosis.

Shoulder involvement in PMR usually affects both sides simultaneously. We described case 2 as presenting with subsequent bilateral involvement. It was difficult to interpret the characteristics of shoulder involvement because the medical record did not describe the state of right shoulder at the time of the surgery on left shoulder. However, because of the similarity of the MRI and arthroscopic findings of both shoulders, we thought that shoulder involvement in case 2 was equivalent on both sides. We suspect that the axial cervical involvement in case 2 was also related to PMR. Interspinous bursitis on MRI or PET/CT studies has been suggested as a potential cause of axial pain in cases of PMR ${ }^{15)}$.

In conclusion, in elderly patients PMR is frequently unrecognized and misdiagnosed as another disease. The clinician should be aware that PMR is associated with shoulder and hip pain, and cerebrovascular accidents. A careful history and meticulous examination are necessary for the correct diagnosis of PMR.

Conflicts of Interest Disclosures: The researchers claim no conflicts of interest.

\section{REFERENCES}

1. Weyand CM, Goronzy JJ. Clinical practice. Giant-cell arteritis and polymyalgia rheumatica. N Engl J Med 2014;371:50-7.

2. Dalkuliç E, Tufan AN, Hafizoğlu E, Hafizoğlu M, Tufan F, Oksuz F, et al. The process from symptom onset to rheumatology clinic in polymyalgia rheumatica. Rheumatol Int 2014;34:1589-92.

3. Nesher G. Neurologic manifestations of giant cell arteritis. Clin Exp Rheumatol 2000;18(4 Suppl 20):S24-6.

4. Kang JH, Sheu JJ, Lin HC. Polymyalgia rheumatica and the risk of stroke: a three-year follow-up study. Cerebrovasc Dis 2011;32:497-503.

5. Hunder GG. Clinical features of GCA/PMR. Clin Exp Rheumatol 2000;18(4 Suppl 20):S6-8.

6. González-Gay MA, Matteson EL, Castañeda S. Polymyalgia rheumatica. Lancet 2017;390:1700-12.

7. Narváez J, Estrada P, López-Vives L, Ricse M, Zacarías A, Heredia S, et al. Prevalence of ischemic complications in patients with giant cell arteritis presenting with apparently isolated polymyalgia rheumatica. Semin Arthritis Rheum 2015;45:32833.

8. Hernández-Rodríguez J, Font C, García-Martínez A, EspígolFrigolé G, Sanmartí R, Cañete JD, et al. Development of ischemic complications in patients with giant cell arteritis presenting with apparently isolated polymyalgia rheumatica: study of a series of 100 patients. Medicine (Baltimore) 2007;86:233-41.

9. Kubota K, Yamashita H, Mimori A. Clinical value of FDG-PET/ CT for the evaluation of rheumatic diseases: rheumatoid arthritis, polymyalgia rheumatica, and relapsing polychondritis. 
Semin Nucl Med 2017;47:408-24.

10. Yamashita H, Kubota K, Takahashi Y, Minaminoto R, Morooka $\mathrm{M}$, Ito $\mathrm{K}$, et al. Whole-body fluorodeoxyglucose positron emission tomography/computed tomography in patients with active polymyalgia rheumatica: evidence for distinctive bursitis and large-vessel vasculitis. Mod Rheumatol 2012;22:705-11.

11. Lariviere D, Benali K, Coustet B, Pasi N, Hyafil F, Klein I, et al. Positron emission tomography and computed tomography angiography for the diagnosis of giant cell arteritis: a real-life prospective study. Medicine (Baltimore) 2016;95:e4146.

12. Koh ES, Lim JY. The management of shoulder pain in the elderly: focusing on clinical characteristics and conservative treatment. J Korean Geriatr Soc 2013;17:1-6.

13. Cho $\mathrm{CH}$, Oh GM. Prognostic factors affecting the clinical outcome of septic arthritis of the shoulder. J Hand Surg Asian Pac Vol 2016;21:339-44.

14. McGonagle D, Pease C, Marzo-Ortega H, O'Connor P, Gibbon W, Emery P. Comparison of extracapsular changes by magnetic resonance imaging in patients with rheumatoid arthritis and polymyalgia rheumatica. J Rheumatol 2001;28:1837-41.

15. Salvarani C, Barozzi L, Cantini F, Niccoli L, Boiardi L, Valentino $\mathrm{M}$, et al. Cervical interspinous bursitis in active polymyalgia rheumatica. Ann Rheum Dis 2008;67:758-61. 